\title{
Ice supersaturation as seen from TOVS
}

\author{
K. Gierens ${ }^{1}$, R. Kohlhepp ${ }^{1}$, P. Spichtinger ${ }^{1}$, and M. Schroedter-Homscheidt ${ }^{2}$ \\ ${ }^{1}$ Deutsches Zentrum für Luft- und Raumfahrt, Institut für Physik der Atmosphäre, Oberpfaffenhofen, Germany \\ ${ }^{2}$ Deutsches Zentrum für Luft- und Raumfahrt, Deutsches Fernerkundungsdatenzentrum, Oberpfaffenhofen, Germany
}

Received: 15 December 2003 - Published in Atmos. Chem. Phys. Discuss.: 16 January 2004

Revised: 17 March 2004 - Accepted: 19 March 2004 - Published: 29 March 2004

\begin{abstract}
We have analysed the upper tropospheric humidity with respect to ice (UTHi) data product obtained from the Television Infrared Observation Satellite (TIROS) Operational Vertical Sounder (TOVS) instrument onboard the National Oceanic and Atmospheric Administration NOAA-14 polar orbiting satellite. While in the production of the official UTHi product values in excess of $100 \%$ are ignored, we do not so - in view of many recent results obtained from a variety of other in-situ and remote sensing instruments showing that ice supersaturation frequently occurs in the upper troposphere. We show that TOVS is able to detect ice supersaturation at the correct locations (however, only in less than one percent of its soundings, presumably because of TOVS's low vertical resolution), and that the supersaturation follows the well known exponential behaviour. We conclude that values of UTHi in excess of saturation should not be considered a measurement error anymore. The similar re-analysis of TOVS data back to 1979 could give important insights into trends of upper tropospheric humidity.
\end{abstract}

\section{Introduction}

Ten years ago Soden and Bretherton (1993) suggested a rather simple method to derive layer-averaged $(300-500 \mathrm{hPa})$ relative humidity (UTH, upper tropospheric humidty) from satellite measured radiances (brightness temperatures) in the water vapour vibration-rotation band $v_{2}$ near $6.7 \mu \mathrm{m}$. The relation is:

$a+b T_{6.7}=\ln \left(\frac{U P_{0}}{\beta \cos \theta}\right)$,

where $a$ and $b$ are constants derived from linear regression, $T_{6.7}$ is the measured brightness temperature in the

Correspondence to: K. Gierens

(klaus.gierens@dlr.de)
$6.7 \mu \mathrm{m}$ channel, and $U$ is $\mathrm{UTH}$ in percent. $P_{0}$ is a dimensionless reference pressure, namely the pressure on the level where the temperature equals $T_{6.7}$ divided by $300 \mathrm{hPa}$. $\beta=\langle d \ln T / d \ln P\rangle$ is a layer-averaged dimensionless lapse rate, and $\theta$ is the satellite view angle.

The quantities $P_{0}$ and $\beta$ in Eq. (1) depend on the temperature profile. While this has been ignored in the original publication of Soden and Bretherton (1993), it was later taken into account by several authors (Soden and Bretherton, 1996; Stephens et al., 1996; Jackson and Bates, 2001), whose work led to a continous improvement of the UTH vs. radiance relationship. The most recent formulation (to our knowledge) is as follows (see Jackson and Bates, 2001):

$$
\begin{aligned}
U & =\frac{1}{P_{H}} \exp \left(a+b T_{12}\right), \quad \text { with } \\
P_{H} & =a^{\prime}+b^{\prime} T_{6} .
\end{aligned}
$$

$P_{H}$ is a new (dimensionless) reference pressure that replaces the old $P_{0}$. This formulation uses the measured (limb corrected) brightness temperatures $T_{6}$ and $T_{12}$ from the High resolution Infrared Radiation Sounder (HIRS/2) channels 6 and 12 , respectively. The constants $\left\{a, b, a^{\prime}, b^{\prime}\right\}$ are regression coefficients determined by Jackson and Bates (2001, their table 2) using the Thermodynamic Initial Guess Retrieval (TIGR-3) dataset (Chaboureau et al., 1998). In the present paper we use the corresponding formulation (i.e. regression coefficients) for the upper tropospheric humidity with respect to ice (UTHi, $U_{i}$ ), i.e.

$$
a=34.161, b=-0.126, a^{\prime}=10.329, b^{\prime}=-0.036,
$$

where the units of the constants are such that $U_{i}$ is in percent, when the brightness temperatures are in Kelvin. The full algorithm also uses the brightness temperature difference between channels HIRS/2 6 and 4: a sounding will be discarded from the UTH retrieval when $T_{6}-T_{4}<20 \mathrm{~K}$, as recommended by Jackson and Bates (2001). The latter are either midlatitude soundings with relatively small temperature lapse rate, 
or soundings in cold air where the measured brightness temperature $T_{12}$ corresponds to a level in the atmosphere below $500 \mathrm{hPa}$ (see Jackson and Bates, 2001).

The UTHi product one can find at the SPARC (Stratospheric Processes And their Role in Climate) WAVAS (WAter Vapour Assessment) data base (ftp://atmos.sparc.sunysb. edu/pub/sparc/wavas/satellite/tovs-etl) has been constrained to values between 0 and $100 \%$ (see the file uth_algorithm.doc on the data base). While the lower of these boundaries obviously makes sense, this is not so for the $100 \%$ boundary. On the contrary, supersaturation with respect to ice does occur relatively frequent in the upper troposphere, as has been shown by measurements from many kinds of instruments (insitu and remote sensing) since the 1940es (Glückauf, 1945; Weickmann, 1945; Brewer, 1946) and in particular since the mid 1990es (e.g. Heymsfield et al., 1998; Jensen et al., 1998; Gierens et al., 1999, 2000; Ovarlez et al., 2000; Vay et al., 2000; Spichtinger et al., 2002, 2003a,b). These measurements are consistent with laboratory results on freezing of aqueous solution droplets in cold (below $-40^{\circ} \mathrm{C}$ ) air (Koop et al., 2000): the homogeneous freezing process, which represents an important formation mechanism of non-convective cirrus clouds, needs substantial supercooling (equivalent to a supersaturation in excess of 40\%) to commence. In regard of these results we had the idea to recalculate the UTHi product without setting values $U_{i}>100 \%$ back to $100 \%$. For this purpose we used a set of TOVS data that was easily accessible; it covers mainly Europe for the period January 1999 to May 2003. We performed this recalculation in order (1) to see whether TOVS detects ice supersaturation at all (which could be difficult in view of the sounder's low vertical resolution), (2) to check where TOVS detects ice supersaturation, and (3) to see whether the values of $U_{i}$ in excess of $100 \%$ follow an exponential distribution (which is generally the case for all other data sets we have analysed before, see Gierens et al., 1999; Spichtinger et al., 2002, 2003a).

The paper is organised as follows: In Sect. 2 we give some details of the data processing prior to application of the UTHi algorithm. Results of our study are presented in Sect. 3 and discussed in Sect. 4. Finally, we draw some conclusions in Sect. 5 .

\section{Data handling}

The UTHi equation was applied to brightness temperature measurements of the HIRS/2 (High resolution Infrared Radiation Sounder) instrument onboard the NOAA-14 satellite. As proposed by Jackson and Bates (2001) HIRS/2 channels no. 6 and 12 (with 13.7 and $6.7 \mu \mathrm{m}$ centre wavelength) were used. HIRS/2 is flown together with the Microwave Sounder Unit (MSU). This combination of HIRS/2, MSU and the Stratospheric Sounding Unit (SSU) is the so called Tele- vision InfraRed Observation Satellite (TIROS) Operational Vertical Sounder (TOVS) instrument suite.

The geographical area is determined by the field of view of the HRPT (High Resolution Picture Transmission) antenna system operated at the German Remote Sensing Data Center (DLR-DFD) in Oberpfaffenhofen, Germany. It covers Europe, the Northern Atlantic as far as parts of Greenland, Northern Africa, the Middle East and reaches as far as western parts of Russia (see Fig. 1).

We investigated daily measurements in the period of January 1999 to May 2003. On average there are six NOAA overpasses per day received in Oberpfaffenhofen and the maximum number is up to seven overpasses per day. The nominal equatorial crossing time of NOAA-14 is 1:30 and 13:30 local solar time. HIRS/2 scans the earth with a scan width of $49.5^{\circ}$ satellite viewing angle and performs a measurement each $1.8^{\circ}$. The HIRS/2 field of view at nadir is $17.4 \mathrm{~km}$ and increases to $58.5 \mathrm{~km}$ at both ends of the scan (NOAA, 1981).

HIRS/2 measurements were processed using the International TOVS Processing Package (ITPP, version 5.20) provided by the Cooperative Institute for Meteorological Satellite Studies (CIMSS) at University of Wisconsin-Madison, United States (ITPP 5.0 User Guide, 1995; Smith et al., 1993, see also http://cimss.ssec.wisc.edu/opsats/polar/iapp/ IAPP.html). The ITPP aims originally at the retrieval of temperature and humidity profiles using the HIRS/2 instrument together with the MSU. In this study it is used as a preprocessing package to derive cloud-cleared brightness measurements as input for the UTHi algorithm.

In a first step raw radiance measurements (counts) are calibrated into brightness temperatures and earth located. The determination of clear sky brightness temperatures needs first a cloud detection using several visible, infrared and microwave tests. As HIRS/2 is flown together with the $\mathrm{Mi}$ crowave Sounder Unit (MSU) and the Advanced Very High Resolution Radiometer (AVHRR), these instruments can be used in synergy in order to detect clouds and correct for cloud contamination in HIRS/2 brightness temperatures (Smith et al., 1993). In order to combine HIRS/2 together with MSU and AVHRR and to facilitate cloud detection, boxes of $3 \times 3$ HIRS/ 2 spots are used. Due to the cloud handling using $3 \times 3$ HIRS/ 2 boxes, brightness temperatures used in this study are also derived for these boxes.

Cloud clearance has been performed using cloud top pressures derived with the $\mathrm{CO}_{2}$ slicing method as used in the ITPP package (Smith and Platt, 1978; Menzel et al., 1983). Only data where cloud top pressures exceed $600 \mathrm{hPa}$ (i.e. layers that do hardly contribute to the signal in HIRS/2 channel 12) are used. Soundings with cloud tops above $600 \mathrm{hPa}$ are discarded. This strategy costs about a third of all our data, but enough data for statistics remain. For TOVS it is also possible to use the $\mathrm{N}^{*}$ algorithm (Smith, 1968; Smith and Woolf, 1976) that produces "cloud free" radiances in cloudy scenes. However, the interpretation of UTHi determined 
from such radiances is not straightforward. Hence, we prefer a stricter cloud clearance even if the statistics might get worse. A check of the influence on our results of varying degrees of strictness in the cloud clearing will be presented later (Sect. 4.3).

ITPP brightness temperature output is not limb-corrected. However, a limb-correction is assumed by the algorithm of Jackson and Bates (2001). Therefore, we have retained for analysis only data where the limb-effects are small, namely data from soundings with viewing angles of less than $33^{\circ}$ from nadir. We have also checked the sensitivity of the results to other cut-off angles. Generally, the influence of the choice of the cut-off angle is small for cut-off values below $33^{\circ}$. The largest influence is on the amount of data for analysis, but neither the spatial distribution nor the humidity distribution (next section) is affected significantly.

\section{Results}

\subsection{Geographical distribution of ice supersaturation}

Figure 1 shows a map (mainly Europe and northern Africa) indicating the relative frequency of supersaturation in the TOVS UTHi data. First one may note that this is rarely more than one percent of the soundings, which is considerably less a fraction than was found previously in MOZAIC and MLS data. The probable reason for this low fraction of soundings with supersaturation is the low vertical resolution of the HIRS/2 channel 12, which is of the order 3 to $5 \mathrm{~km}$. In order that supersaturation is detected in this channel, the supersaturated layer has to be a few $\mathrm{km}$ deep. However, the mean vertical extension of ice supersaturation layers is only half a $\mathrm{km}$ (Spichtinger et al., 2003a); hence very deep ice supersaturation layers that HIRS/2 channel 12 can detect occur rarely. In total we have 3655 records giving ice supersaturation out of 2050,663 , which is a fraction of $0.18 \%$.

Next we note that the highest values occur over the arctic ocean north of Scandinavia and Russia. If we compare this map with a corresponding map derived from MLS data on $215 \mathrm{hPa}$ (Spichtinger et al., 2003b), we do not find much similarity. But keep in mind that HIRS/2 channel 12 sounds the atmosphere mainly between 300 and $500 \mathrm{hPa}$. It is therefore better to compare Fig. 1 with data from lower altitudes. The MLS data on $316 \mathrm{hPa}$ and $464 \mathrm{hPa}$ could in principle be used, however the qualitiy of the relative humidity retrieval on these levels is questionable when the humidity is high, thus we have refrained from producing an ice supersaturation statistics from MLS data on these levels. However, at least one can see in the MLS humidity data (Read et al., 2001, their Plates 10-13) a clear difference between the upper levels (147 and $215 \mathrm{hPa}$ ) where highest humidities are found around the equator, and the lower levels (316 and $464 \mathrm{hPa}$ ) which are relevant for the comparison with TOVS. Here the highest relative humidities are found polewards of

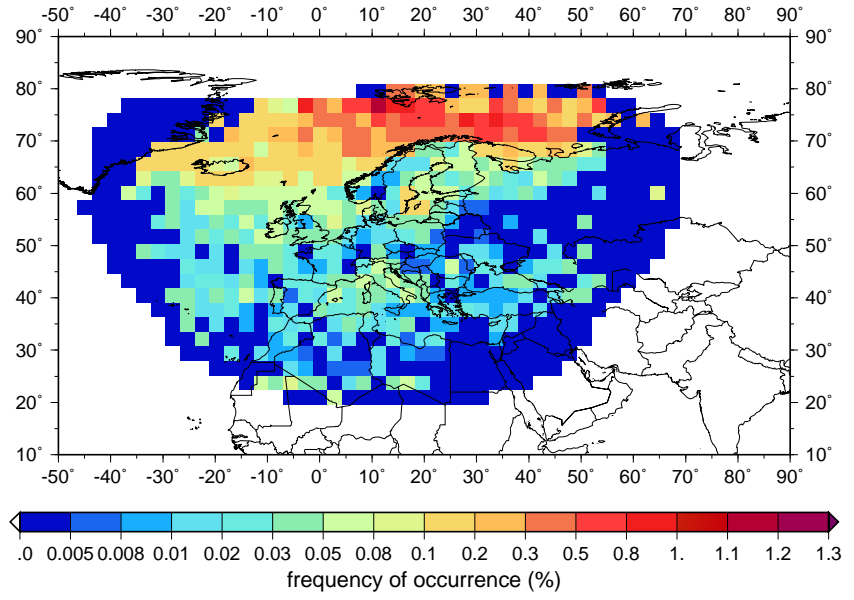

Fig. 1. Map of the frequency of occurrence of ice supersaturation in the TOVS UTHi product obtained from HIRS/2 channel 12 radiance which is sensitive to altitudes between about 300 and $500 \mathrm{hPa}$.

45 to $60^{\circ}$. This is not unexpected since the altitudes of the ice supersaturated layers follow approximately the altitude of the tropopause.

We may also look at humidity climatologies derived from radiosondes. Ross and Elliott (1996, their Fig. 10a) show statistics of relative humidity measured with radiosondes at Sodankyla, Finland. Their figure shows that ice supersaturation occurs more often at $500 \mathrm{hPa}$ than at $300 \mathrm{hPa}$. A similar situation is found at Moosonee, Canada; however this station is not covered in our Fig. 1. Peixoto and Oort (1996, their Fig. 3c) show that at $500 \mathrm{hPa}$ the maxima of annual mean relative humidity $(U>60 \%$, which in winter means almost $\left.U_{i} \geq 100 \%\right)$ are found in the arctic and antarctic latitudes.

Finally, we also consider a proxy, viz. the so-called potential contrail coverage $\left(B_{c}\right)$ derived by Sausen et al. (1998). $B_{c}$ measures the atmosphere's capacity to hold contrails; if aircraft flew everywhere and always the true fractional coverage of contrails would be $B_{c}$. $B_{c}$ can be computed from local temperature and relative humidity using the thermodynamic criterion for contrail formation (see Schumann, 1996). As contrails need ice supersaturation to be persistent, the potential contrail coverage is an appropriate proxy. In Fig. 2 of Sausen et al. (1998) one can see how the maxima of $B_{c}$ are shifted polewards with decreasing altitude: below $300 \mathrm{hPa}$ (i.e. the altitude regime that HIRS/2 channel 12 is sensitive to) the maxima are found poleward of $45^{\circ}$, and with decreasing altitude high values of $B_{c}$ concentrate more and more towards the poles. Thus we conclude that our TOVS map of relative frequency of ice supersaturation is consistent with all these results. 


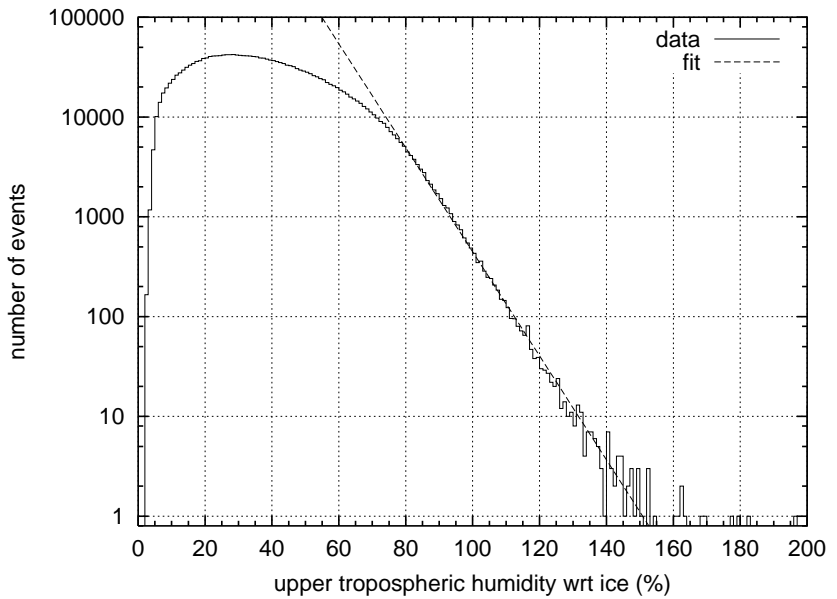

Fig. 2. Non-normalised statistical distribution of UTHi (solid) derived from HIRS/2 channel 12 data via the algorithm of Eqs. (2) and (3), together with an exponential distribution, fitted to the data in the supersaturated range (dashed).

\subsection{Statistical distribution of $U_{i}$ and supersaturation}

The statistical distribution of $U_{i}$, binned into $1 \%$ intervals, from the HIRS/2 channel 12 data is plotted in Fig. 2. Obviously it is possible to fit an exponential function to the data for $U_{i} \geq 80 \%$, which means that the probability to obtain a certain degree of supersaturation $S=U_{i}-100 \%$ decreases exponentially with increasing $S$. This finding is in accord with results from our previous analyses of humidity data from MOZAIC, MLS, and the Lindenberg radiosonde, although these analyses mainly referred to higher altitudes in the troposphere. A significant difference to the earlier analyses is the fact that the TOVS data do not show any sign of a kink in the humidity statistics at ice saturation. We do not currently know the reason for this difference (see discussion).

The slope of the exponential is $\mu=0.12$, implying a mean supersaturation of $8 \%$, which value is close to results from our earlier analyses. The slopes evaluated for single years from 1999 to 2003 show no significant variation.

\section{Discussion}

\subsection{Statistical distribution of $T_{12}$}

We have seen that $S=U_{i}-100 \%$ in the TOVS UTHi product follows an exponential distribution, which result is in line with previous ones obtained from analyses of humidity data from MOZAIC (Gierens et al., 1999), MLS (Spichtinger et al., 2002), and radiosondes (Spichtinger et al., 2003a). Because of the tight relation between $T_{12}$ and $U_{i}$ the question then arises about the underlying statistical distribution of the brightness temperature. This investigation should also be done in view of the results of Kärcher and Haag (2004) and Buehler and Courcoux (2003), who show how natural

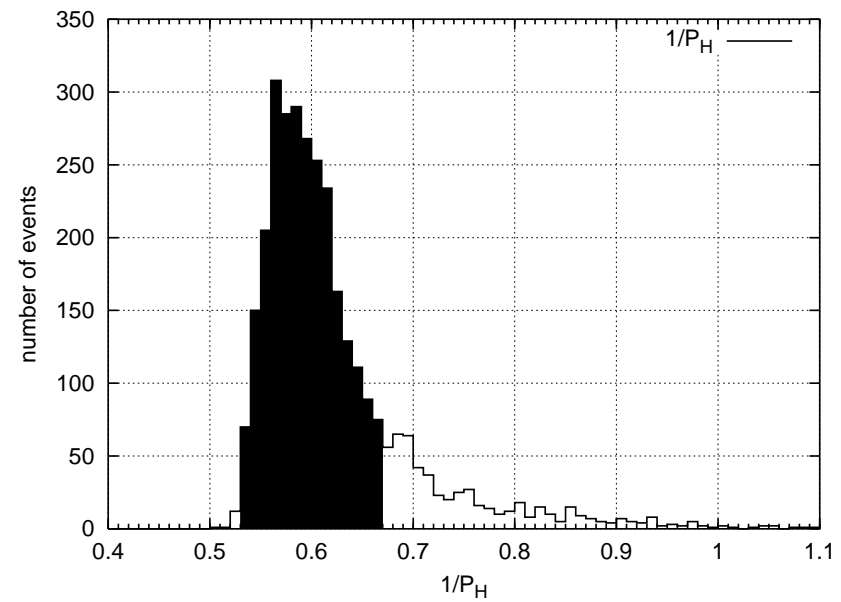

Fig. 3. Non-normalised statistical distribution of the inverse reference pressure $1 / P_{H}$ in ice supersaturated regions. The mean value is at about 0.63 , and $81 \%$ of the data lie in the shaded region between 0.52 and 0.67 .

temperature fluctuations (e.g. the instantaneous temperature distribution within the field of view of a satellite instrument) and temperature retrieval errors (both assumed to be normally distributed) translate into an exponential distribution of supersaturation.

Let us first consider the distribution of the inverse reference pressure $1 / P_{H}$ in the TOVS records indicating supersaturation. This is displayed in Fig. 3. It is a monomodal distribution with the mean value $\left\langle\left(1 / P_{H}\right)\right\rangle=0.63$ and a standard deviation of 0.31 . The variation of $1 / P_{H}$ complicates the derivation of the distribution of the brightness temperatures. Therefore, we first derive the distribution $T_{12}$ under the condition that $1 / P_{H}$ has a given value. The derivation is given in the Appendix.

The probability density of $T_{12}$ for a given value of $1 / P_{H}$, $g_{\tau}(t)\left(\tau=T_{12}-T_{12}^{*}, \tau \leq 0\right)$, is predicted to follow a Gumbel distribution up to the cut-off value $T_{12}^{*}$ that corresponds to ice saturation for the chosen $1 / P_{H}$. Figure 4 shows the TOVS $T_{12}$ data for the range of $1 / P_{H}$ that is shaded in Fig. 3. The corresponding cut-off $T_{12}^{*} \approx 230 \mathrm{~K}$. In the figure the cut-off in the data is less sharp then theory predicts; this is a consequence of using a range of $1 / P_{H}$ instead of an exact value. A smaller $1 / P_{H}$ range would result in a sharper cut-off, but also in noisier data. Yet we dare to state that the agreement between the theoretical distribution and the data is very good.

The distribution of $T_{12}$ with inverse reference pressure whatsoever (Fig. 5) is a bit broader than the one shown in Fig. 4. This is of course the consequence of the variation in the cut-off brightness temperatures. It can be reproduced with the theoretically derived distribution for constant values of $1 / P_{H}$ by computing $g_{\tau}(t)$ for each bin of $1 / P_{H}$ in Fig. 3, and adding them with weighting factors equal to the corresponding number of events in that figure. The result of that exercise is also plotted in Fig. 5; obviously it fits the observed data excellently. 


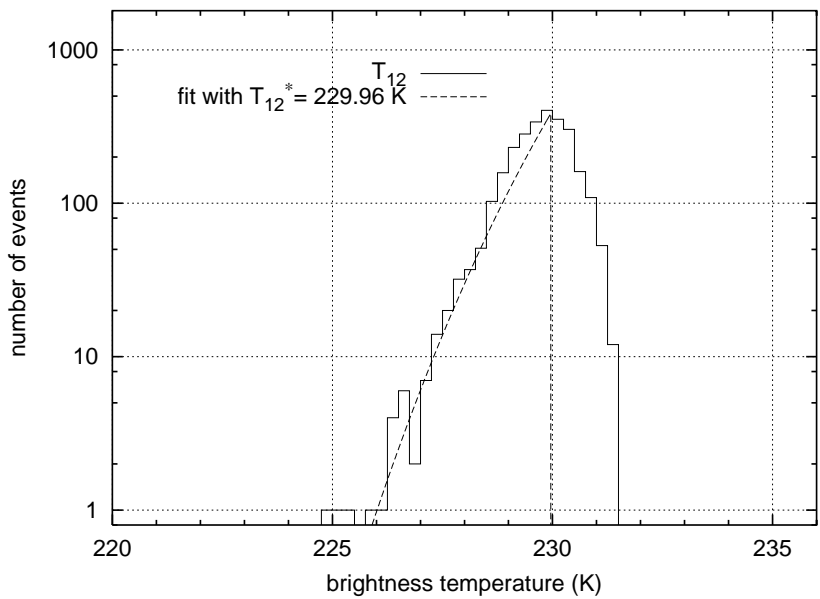

Fig. 4. Non-normalised statistical distribution (solid line) of the brightness temperature in HIRS/2 channel 12, $T_{12}$, for soundings indicating supersaturation $\left(U_{i}>100 \%\right)$, and where the inverse reference pressure is between 0.52 and 0.67 . The broken line is a theoretically derived distribution (normalised to the maximum number of events in the $1 / P_{H}$ distribution of Fig. 3, namely 308 events with $\left.1 / P_{H}=0.56\right)$. It fits the data quite well. It has a sharp cut-off at the indicated value (about $230 \mathrm{~K}$ ). The cut-off in the data is less sharp because the range of $1 / P_{H}$ implies a range of cut-offs. Note that supersaturation increases with decreasing brightness temperature, that is, to the left in the figure.

The brightness temperature in HIRS/2 channel 12 is approximately equal to the atmospheric temperature in that altitude, $\bar{z}$, where the corresponding weighting kernel peaks (where the optical depth at $6.7 \mu \mathrm{m}$, measured from the satellite down into the atmosphere, reaches unity). According to the results above, the temperature distribution at $\bar{z}$, for given $1 / P_{H}$ and in supersaturated regions, should follow a cutoff Gumbel distribution. The question, how the atmosphere could arrange for such a special distribution, naturally arises, but this question reaches beyond the purpose of the present paper. It might be that arguments from extreme value statistics could give an answer, because the Gumbel distribution is one of the three types of extreme value distributions. It is not probable, that this special distribution could result from natural temperature fluctuations or retrieval errors, which both are expected to follow normal (Gaussian) distributions, although they could contribute to the observed distribution. Thus we conclude that the exponential distribution of supersaturation has some deeper origins than simple normal temperature fluctuations, e.g. the random mechanisms described by Gierens et al. (1999). Fortunately, natural temperature fluctuations and Gaussian temperature retrieval errors do not destroy the underlying exponential distribution of supersaturation, they only have an effect on the slope of the exponential. It should be noted that Soden and Bretherton (1993) derived their relation without any anticipated knowledge of the exponential distribution of ice supersaturation which was

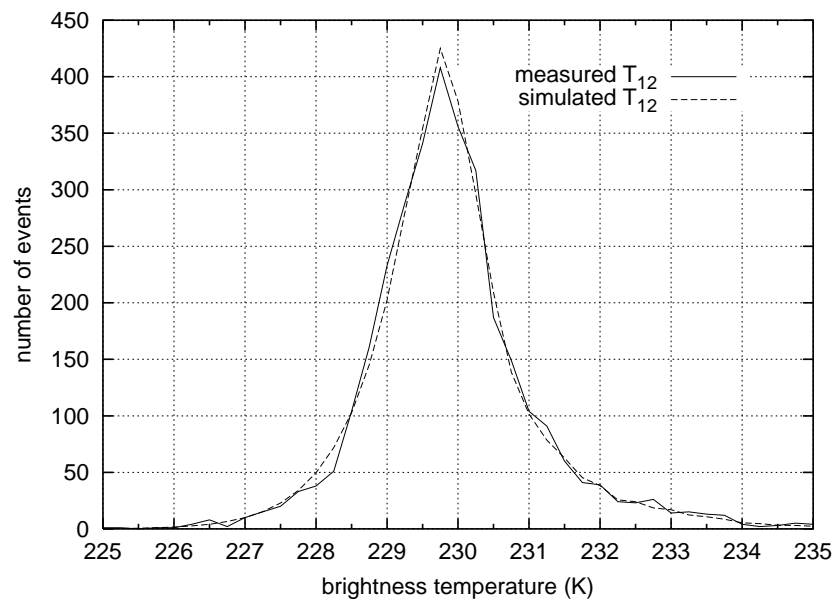

Fig. 5. As Fig. 4, however for the full range of $1 / P_{H}$. The excellent agreement between data and fit may be noted.

found later by Gierens et al. (1999), and their linear relation between UTHi and $e^{T_{12}}$ is the only way to translate the peculiar distribution of $T_{12}$ or $T(\bar{z})$ into the exponential distribution of supersaturation.

\subsection{The missing kink and the regression coefficients}

In previous analyses of statistics of relative humidity the tropospheric distributions always showed a kink at saturation which is missing here. Usually the kink is interpreted to be the consequence of the fact that cloud processes can act above, but not below saturation. Such a kink must be present as soon as there is cloud formation in the probed part of the atmosphere, even when the best cloud clearing algorithm in the world would be applied to the data. A kink is usually absent when no clouds can form; e.g. stratospheric data, at least when they are obtained from some kilometres above the tropopause (e.g. MLS on $147 \mathrm{hPa}$ in the extratropics) usually do not display a kink at saturation. Of course, in the regions probed by HIRS/2 channel 12 there is sufficient cloudiness for a kink to form. Hence, there must be another explanation. There is a soft kink at $U_{i} \approx 80 \%$, which might suggest a scaling problem; however, the UTH algorithm has been tested and continuously improved for the recent ten years, so we are convinced that there is no scaling error.

Another possibility one might think of is that the kink gets lost or shifted to $80 \%$ because of the low vertical resolution. As UTHi is an average relative humidity over a column of air a few kilometres thick it cannot be excluded that cloud processes in one part of the column influence the obtained mean value even when that mean is below saturation (remember that the mere possibility of cloud formation changes the slope of the distribution even with cloud clearing employed on the data). In order to check this possibility we have tested the influence of a quasi HIRS/2 channel 12 weighting function on radiosonde humidity measurements that have been 


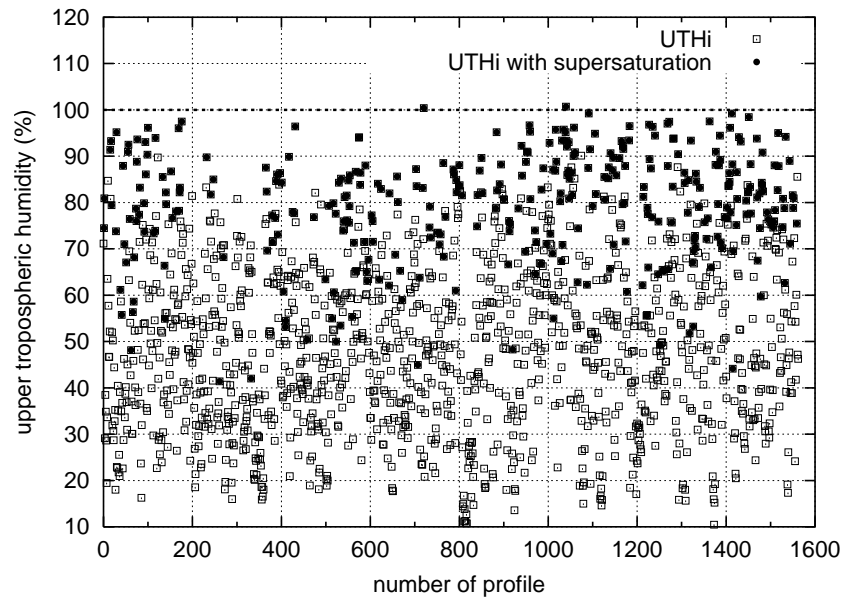

Fig. 6. UTHi as vertically averaged relative humidity with respect to ice from data of radiosonde soundings from the meteorological observatory Lindenberg. The averaging procedure is given in Eqs. (4) and (5). Black dots mark soundings where an at least $1 \mathrm{~km}$ thick ice supersaturation layer was at an altitude where $K \geq 0.075$.

used recently for analysis of ice supersaturation in the upper troposphere (Spichtinger et al., 2003a). These are data from the German meteorological observatory at Lindenberg $\left(52.22^{\circ} \mathrm{N}, 14.12^{\circ} \mathrm{E}\right)$ obtained with a Vaisala RS80A instrument and with corrections and careful gauging applied. Our radiosonde data set comprises mostly 4 soundings per day from February 2000 to April 2001, about 1600 profiles. We have applied the following weighting kernel to the data (the connoisseur will notice that this - coincidentally - is also a Gumbel distribution):

$K(z-\bar{z} ; H)=H^{-1} e^{-(z-\bar{z}) / H} \exp \left(-e^{-(z-\bar{z}) / H}\right)$,

with scale height $H=2 \mathrm{~km}$ for a typical exponential water vapour concentration profile. The peak of the weighting kernel is at $\bar{z}$, which is determined for each radiosonde profile as that altitude where the optical depth (measured downwards from the highest point the sonde arrives at) at $6.7 \mu \mathrm{m}$ reaches unity. The optical depth has been computed using eqns. (8) and (9) of Stephens et al. (1996). Using this kernel, we compute for each profile of relative humidity with respect to ice, $R H_{i}(z)$, the following average:

$U_{i}=\int_{0}^{\infty} R H_{i}(z) K(z-\bar{z} ; H) d z$.

The results are plotted in Fig. 6, which shows that the vast majority of values is below saturation, although there is a mean frequency of occurrence of ice supersaturation cases (true ISSRs and cirrus clouds) of $28 \%$ in the radiosonde data (Spichtinger et al., 2003a). The radiosonde profiles where an at least $1 \mathrm{~km}$ thick ice supersaturation layer was at an altitude where $K \geq 0.075$ (comprising about $80 \%$ of the total weight) are marked as black dots in the figure. Although these points are more concentrated to the saturation line, only few of these

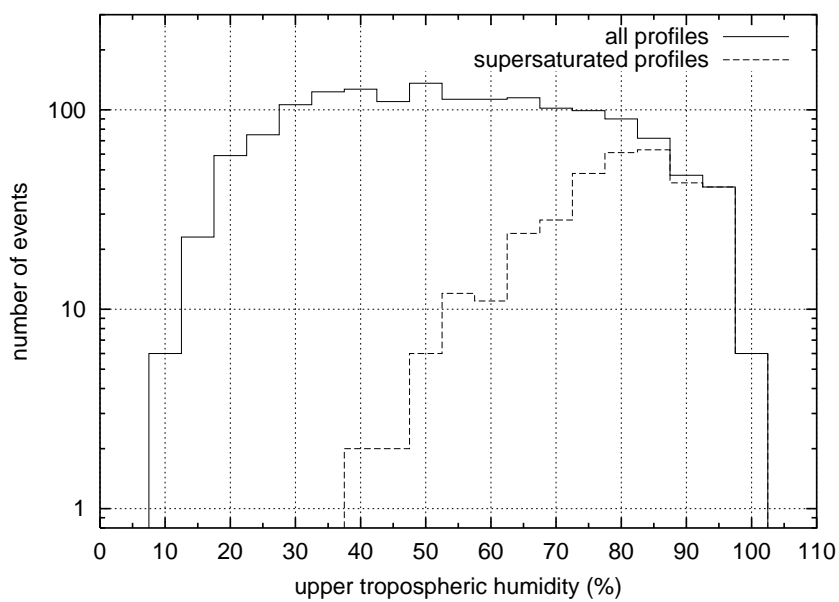

Fig. 7. Histogram with classes of 5\% width of the results presented in Fig. 6. The dashed curve corresponds to the black dots in that Figure. The strong decrease of the number of events beyond UTHi of $80-90 \%$ can be noticed.

actually are above $100 \%$. Obviously the coarse vertical averaging pretends much less ice supersaturation than actually present, in this case only $0.2 \%$, which is similar to the fraction of supersaturation cases in the analysed TOVS data. It can also be noticed that the density of points decreases already above $80-90 \%$ (see Fig. 7). Hence we think at the moment that the coarse vertical resolution of the TOVS data is the most plausible explanation for the shifted (or missing) kink.

The empirical regression coefficients $a$ and $b$ have been determined under the assumption that soundings giving $U_{i} \geq 100 \%$ are erroneous. It is possible and probable that the regression coefficients would change somewhat when supersaturation cases would be considered, and when additionally ice supersaturation in the atmospheric profiles of the Thermodynamic Initial Guess Retrieval (TIGR) dataset would not be reset to saturation for the forward radiative transfer calculations. Yet, this question cannot be answered here.

\subsection{Influence of retrieval errors}

A weak point of the UTH algorithm is the fact that retrieval errors of the HIRS/2 channel 12 brightness temperature scale linearly with the relative uncertainty in UTH (Soden et al., 2000). This implies that the largest uncertainties of UTH occur just in the ice supersaturated situations. The regression parameter $b=-0.126$ means that at saturation a $1 \mathrm{~K} \mathrm{er}-$ ror in $T_{12}$ (and $T_{6}$ ) means an error of about $13 \%$ in UTHi, and still larger errors under supersaturation conditions. Jackson and Bates (2001, their Fig. 13) show UTH sensitivity curves for systematic difference between modelled and observed brightness temperatures. In this figure the sensitivity $\left(\left|d U / d T_{12}\right|\right)$ increases strongly with decreasing brightness temperature, but the sensitivity is weaker for higher values 
of $P_{H}$. For us this means that we are unfortunately at the high sensitivity end (since $T_{12}$ is typically below $235 \mathrm{~K}$ in the supersaturated cases), but fortunately, in their figure, on the curve with the least steep increase of sensitivity with decreasing $T_{12}$ (since $P_{H}$ is typically 1.7, see Fig. 3).

We have tested the influence of random normally distributed errors in both $T_{6}$ and $T_{12}$ with standard deviation of $1 \mathrm{~K}$. The corresponding noise levels in these channels (for NOAA-7) are 0.54 and $0.84 \mathrm{~K}$, respectively (Eyre, 1987), including radiometric noise as well as other errors. Hence we do a worst case estimate here. Each brightness temperature was randomly disturbed and the corresponding UTHi product was re-computed. This procedure again led to an exponential distribution of $S$, the degree of supersaturation, however, with a slightly reduced slope of $\mu=0.1$, which translates into a slightly larger mean supersaturation of $10 \%$. Effects of the limb-correction are smaller than this effect of random temperature errors. Regression noise (i.e. the effect of atmospheric parameters that are not accounted for in the simple relationship of Eqs. 2 and 3) could in principle lead to errors in the statistics of supersaturation presented above. However, the analysis in the previous section showed that TOVS data rather suffer from an underestimation of the number of supersaturation events than from the contrary. Hence, we can safely assume that our false alarm rate (i.e. the retrieval indicates supersaturation where actually none is) is very low (if not zero). When the UTHi retrieval was applied to TIGR-3 profiles with any supersaturation in the profiles removed, no value of UTHi was in excess of saturation. A test of various cloud clearance strategies (cut-offs of 600 and $500 \mathrm{hPa}$, no cloud clearance at all) shows no noticable effect on the slope of the humidity distribution. The most probable explanation for this "miracle" is that by the $T_{6}-T_{4}$ criterion (see text after Eq. 3) already most ( $84.3 \%$ in our case) of the data contaminated by high clouds (tops above $600 \mathrm{hPa}$ ) are discarded from the further analysis. We conclude, that although it is not advisable to trust a single value of UTHi, in particular in situations characterised by a wet profile, it is still possible to trust the general characteristics of the humidity statistics one can derive. In particular, the exponential nature of the humidity distribution turns out a robust feature.

\section{Conclusions}

In the present paper we have applied the most recent formulation of the TOVS UTHi algorithm (Jackson and Bates, 2001) to a subset of NOAA-14 HIRS/2 channel 12 data mainly covering Europe and North Africa for the period January 1999 to May 2003. Doing this, we have retained measurements that give UTHi in excess of ice-saturation, which are normally ignored when the UTHi product is produced. We retained these measurements because of the many results now available that indicate the presence of large supersaturated regions in the upper troposphere. Our aim here was threefold:
1. to see whether TOVS, having a vertical resolution (say, $3 \mathrm{~km}$ ) that is much coarser than a typical vertical extension of ISSRs (Spichtinger et al., 2003a, about $0.5 \mathrm{~km}$ ) is able at all to detect ISSRs. Indeed, TOVS detects ISSRs, however, less than half a percent of the TOVS data indicate ice supersaturation. This is much smaller a fraction than obtained from other data sets, in particular from the in-situ measurements of MOZAIC (about $15 \%$ ). Obviously, with TOVS one can only detect very vertically extended ISSRs.

2. to see where TOVS detects ISSRs. The spatial distribution of ISSRs found by TOVS is typical for the altitude range where HIRS/2 channel 12 is sensitive to, i.e. 300$500 \mathrm{hPa}$. In that range higher degrees of humidity are found more in the northern latitudes than over southern Europe and Africa. The picture that TOVS furnishes for ISSRs is consistent with other humidity data sets for 300-500 hPa.

3. to see whether the supersaturation statistics for UTHi follows an exponential distribution. This was indeed the case, and is sofar consistent with results from MOZAIC, MLS, and the Lindenberg radiosonde analyses. However, other tropospheric data sets show regularly a kink in the slope of the distribution at saturation, which is missing in the TOVS UTHi data, or, more precisely, shifted to $80 \%$ UTHi. At the moment we believe that this is an effect of the coarse vertical resolution of the nadir sounding TOVS. At least, a simple test using true radiosonde data gave a result that was consistent with our suggestion.

We have shown, that the underlying distribution of the channel 12 radiance, or the corresponding brightness temperature, follows a rather peculiar distribution (conditioned on a certain value of $1 / P_{H}$ and on $S>0$ ), namely a cut-off Gumbel distribution. Such a peculiar distribution, which must be the distribution of the atmospheric temperature at the altitude where the channel 12 kernel function peaks, cannot be derived from Gaussian natural temperature fluctuations or temperature retrieval errors. Thus, attempts to explain the observed exponential distribution of supersaturation with such Gaussian temperature fluctuations and/or retrieval errors do not apply here. We conclude from this that there are deeper origins of the exponential distribution of supersaturation than just Gaussian temperature fluctuations and retrieval errors, for example the memoryless source- and sink processes suggested by Gierens et al. (1999).

Finally we conclude that TOVS soundings giving UTHi in excess of saturation are not neccessarily erroneous and should be retained in the UTH products. New investigations should be performed to determine the regression coefficients for the supersaturated cases, because they may differ from the published ones. At least, ice supersaturation in the profiles should not be reset to saturation for forward 
radiative transfer calculations. Also the TOVS community should ponder about the possibility to include atmospheric profiles based on reliable humidity measurements in the cold upper troposphere (including ice supersaturation) into their Thermodynamic Initial Guess Retrieval (TIGR) data sets. Such profiles are available at least from the Lindenberg observatory of the German Weather Service (Spichtinger et al., 2003a)

The shortcoming of TOVS regarding its vertical resolution can be overcome in the near future with the advent of the next generation of infrared sounders with high spectral resolution, as AIRS (Atmospheric InfraRed Sounder, already in orbit), or IASI (Infrared Atmospheric Sounding Interferometer, to be launched in 2005). These instruments cover the $\mathrm{H}_{2} \mathrm{O} \nu_{2}$ band with many $(>100)$ channels. The corresponding kernel functions will peak in different altitudes and the information contained in the many channels, e.g. on UTHi, can be deconvoluted to provide more than one independent piece of information about UTHi. The detection efficiency for ISSRs will increase with the improvement of the sounder's vertical resolution.

However, although TOVS sees only a small fraction of the ISSRs present in the upper troposphere, it is still worthwhile to analyse ice supersaturation statistics with TOVS, because this is a long-term data set. Its exploitation would enable us for the first time to see whether the amount of ice supersaturation in the upper troposphere is sensitive to a changing climate.

\section{Appendix: Mathematical details}

As we saw, the supersaturation $S=U_{i}-100 \%$ is distributed exponentially, i.e. its cumulative distribution function is $F_{S}(s)=1-\exp (-\mu s)$, with a constant $\mu=0.12$ (determined from the data). The relation between $S$ and the brightness temperature $T_{12}$ is of the form $S=A\left(e^{b T_{12}}-e^{b T_{12}^{*}}\right)$ where $T_{12}^{*}$ is the brightness temperature that yields saturation, i.e. $U_{i}^{*}=100 \% . A$ is an abbreviation for $e^{a} / P_{H}$. In the following we consider only cases with a fixed value of $1 / P_{H}$ in order to simplify the derivation. The relation between $S$ and $T_{12}$ can be rearranged into

$S=A^{*}\left(e^{b \tau}-1\right)$

with $A^{*}=A e^{b T_{12}^{*}}$ and $\tau=T_{12}-T_{12}^{*}$. Resolving for $\tau$ yields

$\tau=\ln \left(1+S / A^{*}\right) / b$.

Please note that $\tau \leq 0$ since $b$ is negative, hence $b \tau \geq 0$.

Now we compute the cumulative distribution function for $\tau, G_{\tau}(t)(\operatorname{Pr}\{\cdot\}$ means probability of the event given in braces):

$$
\begin{aligned}
G_{\tau}(t) & =\operatorname{Pr}\{\tau \leq t\} \\
& =\operatorname{Pr}\left\{\ln \left(1+S / A^{*}\right) / b \leq t\right\} \\
& =\operatorname{Pr}\left\{\ln \left(1+S / A^{*}\right) \geq b t\right\}
\end{aligned}
$$

$$
\begin{aligned}
& =\operatorname{Pr}\left\{S \geq A^{*}\left(e^{b t}-1\right)\right\} \\
& =1-F_{S}\left[A^{*}\left(e^{b t}-1\right)\right] .
\end{aligned}
$$

Note that the direction of the inequality is changed from the 2 nd to the 3 rd line above because the negative $b$ is brought to the rhs. Inserting $F_{S}$ yields

$G_{\tau}(t)=\exp \left[-\mu A^{*}\left(e^{b t}-1\right)\right]$.

The corresponding probability density, $g_{\tau}(t)$ is the derivative of $G_{\tau}$ with respect to $t$, viz.

$g_{\tau}(t)=-\mu A^{*} b e^{\mu A^{*}} \cdot e^{b t} \cdot \exp \left[-\mu A^{*} e^{b t}\right]$,

with $t \leq 0$. The shape of this distribution is that of a Gumbel distribution apart from the cut-off at zero. As it should be, the integral $\int_{-\infty}^{0} g_{\tau}(t) d t=1$.

Gumbel distributions occur frequently in extreme value statistics. The general form of the probability density function of a Gumbel distributed random variable $X$ is as follows:

$g_{X}(x)=\frac{1}{\alpha} \exp \left(\frac{x-x_{0}}{\alpha}\right) \exp \left(-\exp \left(\frac{x-x_{0}}{\alpha}\right)\right)$

where $x_{0}$ is the location parameter and $\alpha$ is the scale parameter.

Acknowledgements. We thank R. Büll for fruitful discussions and two anonymous reviewers for their useful comments.

Edited by: M. G. Lawrence

\section{References}

Brewer, A. W.: Condensation trails, Weather, 1, 34-40, 1946.

Buehler, S. A. and Courcoux, N.: The impact of temperature errors on perceived humidity supersaturation, Geophys. Res. Lett., 30(14), 1759, doi:10.1029/2003GL017691, 2003.

Chaboureau, J.-P., Chédin, A., and Scott, N.A.: Remote sensing of the vertical distribution of atmospheric water vapor from the TOVS observations: Method and validation, J. Geophys. Res., 103, 8743-8752, 1998.

Eyre, J. R.: On systematic errors in satellite sounding products and their climatological mean values, Q. J. Roy. Meteorol. Soc., 113, 279-292, 1987.

Gierens, K., Schumann, U., Helten, M., Smit, H. G. J., and Marenco, A.: A distribution law for relative humidity in the upper troposphere and lower stratosphere derived from three years of MOZAIC measurements, Ann. Geophys. 17, 12181226, 1999.

Gierens, K., Schumann, U., Helten, M., Smit, H. G. J. and Wang, P.-H.: Ice-supersaturated regions and sub visible cirrus in the northern midlatitude upper troposphere, J. Geophys. Res., 105, 22 743-22 754, 2000.

Glückauf, E.: Notes on upper air hygrometry - II: On the humidity in the stratosphere, Q. J. R. Meteorol. Soc., 71, 110-112, 1945.

Heymsfield, A. J., Miloshevich, L. M., Twohy, C., Sachse, G., and Oltmans, S.: Upper-tropospheric relative humidity observations and implications for cirrus ice formation, Geophys. Res. Lett., 25, 1343-1346, 1998. 
ITPP 5.0 User Guide, Cooperative Institute for Meteorological Satellite Studies (CIMSS), University of Wisconsin-Madison, June 1995 (available via ITPP website http://cimss.ssec.wisc. edu/opsats/polar/iapp/IAPP.html).

Jackson, D. L. and Bates, J. J.: Upper tropospheric humidity algorithm assessment, J. Geophys. Res., 106, 32 259-32 270, 2001.

Jensen, E. J., Toon, O. B., Tabazadeh, A., Sachse, G. W., Anderson, B. E., Chan, K. R., Twohy, C. W., Gandrud, B., Aulenbach, S. M., Heymsfield, A., Hallett, J., and Gary, B.: Ice nucleation processes in upper tropospheric wave-clouds during SUCCESS, Geophys. Res. Lett., 25, 1363-1366, 1998.

Kärcher, B. and Haag, W.: Factors controlling upper tropospheric relative humidity, Ann. Geophys., 22, 705-715, 2004.

Koop, T., Luo, B., Tsias, A., and Peter, T.: Water activity as the determinant for homogeneous ice nucleation in aqueous solutions, Nature, 406, 611-614, 2000.

Menzel, W. P., Smith, W. L. and Stewert, T. R.: Improved cloud motion wind vector and altitude assignment using VAS, J. Appl. Meteor., 22, 377-384, 1983.

NOAA Technical Report NESS 83, Atmospheric Sounding Users Guide, 1981.

Ovarlez, J., van Velthoven, P., Sachse, G., Vay, S., Schlager, H., and Ovarlez, H.: Comparison of water vapor measurements from POLINAT 2 with ECMWF analyses in high humidity conditions, J. Geophys. Res., 105, 3737-3744, 2000.

Peixoto, J. P. and Oort, A. H.: The climatology of relative humidity in the atmosphere, J. Climate, 9, 3443-3463, 1996.

Read, W. G., Waters, J. W., Wu, D. L., Stone, E. M., Shippony, Z., Smedley, A. C., Smallcomb, C. C., Oltmans, S., Kley, D., Smit, H. G. J., Mergenthaler, J. L., and Karki, M. K.: UARS MLS Upper Tropospheric Humidity Measurement: Method and Validation, J. Geophys. Res., 106, 32 207-32 258, 2001.

Ross, R. J. and Elliott, W. P.: Tropospheric precipitable water: A radiosonde-based climatology, NOAA Technical Memorandum ERL ARL-219, 132 pp., 1996.

Sausen, R., Gierens, K., Ponater, M., and Schumann, U.: A diagnostic study of the global distribution of contrails part I: Present day climate, Theor. Appl. Climatol., 61, 127-141, 1998.

Schumann, U.: On conditions for contrail formation from aircraft exhausts, Meteorol. Z., N. F. 5, 4-23, 1996.

Smith, W. L.: An improved method for calculating tropospheric temperature and moisture profiles from satellite radiometer measurements, Monthly Weather Rev., 96, 387-396, 1968.
Smith, W. L. and Woolf, H. M.: The use of eigenvectors of statistical covariance matrices for interpreting satellite sounding radiometer observations, J. Atmos. Sci, 33, 1127-1140, 1976.

Smith, W. L. and Platt, C. M. R.: Comparison of satellite-deduced cloud heights with indications from radiosonde and ground based laser measurements, J. Appl. Meteor., 17, 1796-1802, 1978.

Smith, W. L., Woolf, H. M., Nieman, S. J. and Achtor, T. H.: ITPP-5 - The use of AVHRR and TIGR in TOVS Data Processing, Technical Proceedings, 7th International TOVS Study Conference, pp 443-453, Feb. 1993.

Soden, B. J. and Bretherton, F. P.: Upper tropospheric relative humidity from the GOES $6.7 \mu \mathrm{m}$ channel: Method and climatology for July 1987, J. Geophys. Res., 98, 16 669-16 688, 1993

Soden, B. J. and Bretherton, F. P.: Interpretation of TOVS water vapor radiances in terms of layer-average relative humidities: Method and climatology for the upper, middle, and lower troposphere, J. Geophys. Res., 101, 9333-9343, 1996.

Soden, B., Tjemkes, S., Schmetz, J., Saunders, R., Bates, J., Ellingson, B., Engelen, R., Garand, L., Jackson, D., Jedlovec, G., Kleespies, T., Randel, D., Rayer, P., Salathe, E., Schwarzkopf, D., Scott, N., Sohn, B., de Souza-Machado, S., Strow, L., Tobin, D., Turner, D., van Delst, P., and Wehr, T.: An intercomparison of radiation codes for retrieving upper-tropospheric humidity in the 6.3- $\mu \mathrm{m}$ band: A report from the first GVaP workshop, Bull. Amer. Meteorol. Soc., 81(4), 797-808, 2000.

Spichtinger, P., Gierens, K., and Read, W.: The statistical distribution law of relative humidity in the global tropopause region, Meteorol. Z., 11, 83-88, 2002.

Spichtinger, P., Gierens, K., Leiterer, U., and Dier, H.: Ice supersaturation in the tropopause region over Lindenberg, Germany, Meteorol. Z., 12, 143-156, 2003a.

Spichtinger, P., Gierens, K., Read, W.: The global distribution of ice-supersaturated regions as seen by the microwave limb sounder, Q. J. Roy. Meteorol. Soc., 129, 3391-3410, 2003b.

Stephens, G. L., Jackson, D. L., and Wittmeyer, I.: Global observations of upper-tropospheric water vapor derived from TOVS radiance data, J. Clim., 9, 305-326, 1996.

Vay, S. A., Anderson, B. E., Jensen, E. J., Sachse, G. W., Ovarlez, J., Gregory, G. L., Nolf, S. R., Podolske, J. R., Slate, T. A., and Sorenson, C. E.: Tropospheric water vapor measurements over the North Atlantic during the Subsonic Assessment Ozone and Nitrogen Oxide Experiment (SONEX), J. Geophys. Res., 105, 3745-3756, 2000.

Weickmann, H.: Formen und Bildung atmosphärischer Eiskristalle, Beitr. Physik der freien Atmosphäre, 28, 12-52, 1945. 